\title{
SCORES, INEQUALITIES AND REGULAR HYPERTOURNAMENTS
}

\author{
Muhammad Ali Khan, S. Pirzada and Koko K. Kayibi
}

\begin{abstract}
A $k$-hypertournament is a complete $k$-hypergraph with each $k$-edge endowed with an orientation, that is, a linear arrangement of the vertices contained in the edge. In a $k$ hypertournament, the score $s_{i}$ (losing score $r_{i}$ ) of a vertex $v_{i}$ is the number of edges containing $v_{i}$ in which $v_{i}$ is not the last element (in which $v_{i}$ is the last element). In this paper we obtain inequalities involving powers of scores and losing scores by using classical results from mathematical analysis (such as Hölder's inequality) and show that equality holds if and only if the hypertournament is regular. We then use these inequalities to give a short proof of a result on the existence of regular hypertournaments. We also obtain an upper bound on the number of directed paths of length 2 in tournaments and hypertournaments, prove that the bound is sharp and that it is realized by regular hypertournaments.
\end{abstract}

Mathematics subject classification (2010): 05C20, 05 C65.

Keywords and phrases: tournament, hypertournament, score, losing score, Hölder's inequality, Minkowski's inequality, power mean, inner product, regular hypertournament, Mahler's inequality.

\section{REFERENCES}

[1] C. M. Bang And H. Sharp JR., Score vectors of tournaments, J. Combin. Theory Ser. B 26, 1 (1979), 81-84.

[2] C. Berge, Graphs and Hypergraphs, North-Holland Mathematical Library 6, North-Holland Publishing Co., Amsterdam, London, 1976.

[3] D. BRCANOV AND V. Petrovic, Kings in multipartite tournaments and hypertournaments, Numerical analysis and applied mathematics: International Conference on Numerical Analysis and Applied Mathematics 2009: Volume 1 and Volume 2, AIP Conference Proceedings, 2009, 1255-1257.

[4] R. A. BRUALDI AND J. SHEN, Landau's inequalities for tournament scores and a short proof of a theorem on transitive sub-tournaments, J. Graph Theory 38, 4 (2001), 244-254.

[5] A. IVANYI, Reconstruction of complete interval tournaments, Acta Univ. Sapientiae Informatica 1, 1 (2009), 71-88.

[6] K. K. KAYIBI, M. A. KhAn AND S. PIRZADA, Uniform sampling of $k$-hypertournaments, communicated.

[7] Y. KoH And S. ReE, Score sequences of hypertournament matrices, J. Korea Soc. Math. Educ. Ser. B: Pure and Appl. Math. 8, 2 (2001), 185-191.

[8] Y. KoH AND S. ReE, On k-hypertournament matrices, Linear Algebra Appl. 373, November (2003), $183-195$.

[9] H. G. LANDAU, On dominance relations and the structure of animal societies. III. The condition for a score structure, Bull. Math. Biophys. 15 (1953), 143-148.

[10] S. PiRZADA, Degree sequences of k-multi-hypertournaments, Appl. Math. J. Chinese Univ. Ser. B 24, 3 (2009), 350-354.

[11] S. Pirzada, T. A. Chishti And T. A. NAIKoo, Score sequences in $[h-k]$-bipartite hypertournaments, Discrete Math. Appl. 19, 3 (2009), 321-328.

[12] S. PirZada, T. A. NAikoo AND G. Zhou, Score lists in tripartite hypertournaments, Graphs Combin. 23, 4 (2007), 445-454.

[13] S. PirZada And G. Zhou, Score lists in $(h, k)$-bipartite hypertournaments, Appl. Math. J. Chinese Univ. Ser. B 22, 4 (2007), 485-489. 
[14] S. PiRzAda AND G. Zhou, Score sequences in oriented k-hypergraphs, Eur. J. Pure Appl. Math. 1, 3 (2008), 10-20.

[15] S. PiRZADA AND G. Zhou, On k-hypertournament losing scores, Acta Univ. Sapientiae, Informatica 2, 1 (2010), 5-9.

[16] C. WANG AND G. ZHou, Note on the degree sequences of $k$-hypertournaments, Discrete Math. 308, 11 (2008), 2292-2296.

[17] G. Zhou And S. Pirzada, Degree sequences in oriented $k$-hypergraphs, J. Applied Mathematics and Computing 27, 1-2 (2008), 149-158.

[18] G. ZHOU, T. YAO AND K. ZHANG, On score sequences of $k$-hypertournaments, European J. Combin. 21, 8 (2000), 993-1000. 